\title{
A NOVEL TWO-PORT 6T CMOS SRAM CELL STRUCTURE FOR LOW-VOLTAGE VLSI SRAM WITH SINGLE-BIT-LINE SIMULTANEOUS READ-AND-WRITE ACCESS (SBLSRWA) CAPABILITY
}

\author{
B. T. Wang and James B. Kuo \\ Rm. 338, Dept. of Electrical Eng., National Taiwan University \\ Roosevelt Rd., Sec. 4, Taipei, Taiwan 106-17 \\ Fax:886-2-2363-6893, Telephone:886-2-2363-5251x338 \\ Email:jbkuo@cc.ee.ntu.edu.tw
}

\begin{abstract}
This paper reports a two-port 6T CMOS SRAM cell structure for low-voltage VLSI SRAM with single-bit-line simultaneous read-and-write access (SBLSRWA) capability. With a unique structure by connecting the source terminal of an NMOS device in the SRAM cell to the write word line, this SRAM cell can be used to provide SBLSRWA capability for IV two-port VLSI SRAM as verified by SPICE results.
\end{abstract}

\section{Summary}

\section{Introduction}

In a conventional SRAM cell as shown in Fig. 1, double bit lines have been used for read and write accesses - read and write operations are done via the same pair of bit lines. Therefore, while designing two-port memory IC with simultaneous read and write capability, two more pass transistors and an extra pair of bit lines are needed to be incorporated into the memory cell. As a result, the size of the memory cell is increased substantially. If the memory cell structure can be simplified to provide the operation with only one bit line for read and the other bit line for write, the size of this memory cell can be reduced a lot for implementing two- port VLSI SRAM.

The difficulty of using the conventional SRAM cell with single-bit-line write access can be perceived from Fig. 2 . As shown in Fig. 2, if logic-1 is to be written from the left bit line WBL via the pass transistor $\mathrm{M}_{\mathrm{N} 1}$ into the left side of the memory cell (node $\mathrm{n} 1$ ), where logic- 0 is originally stored. During the write-in operation, the voltage at node $\mathrm{n} l$ cannot be raised to exceed $V_{d d}-V_{T N}$ by the write bit line WBL, where $V_{d d}$ is the power supply voltage and $V_{T N}$ is the threshold voltage of the pass transistor $\mathrm{M}_{\mathrm{N} 1}$. In addition, since the ratioed-logic structure, which is made of the NMOS devices $M_{N 1}$ and $M_{N 3}$ in the memory cell and the PMOS device $M_{\mathrm{P} 3}$, which is the driver of the bit line WBL, plus the fact that the electron mobility of the NMOS devices

This work is supported under R.O.C. National Science Council Contracts \#88-2215-E002-033 \& \#88-2622-E002-028.

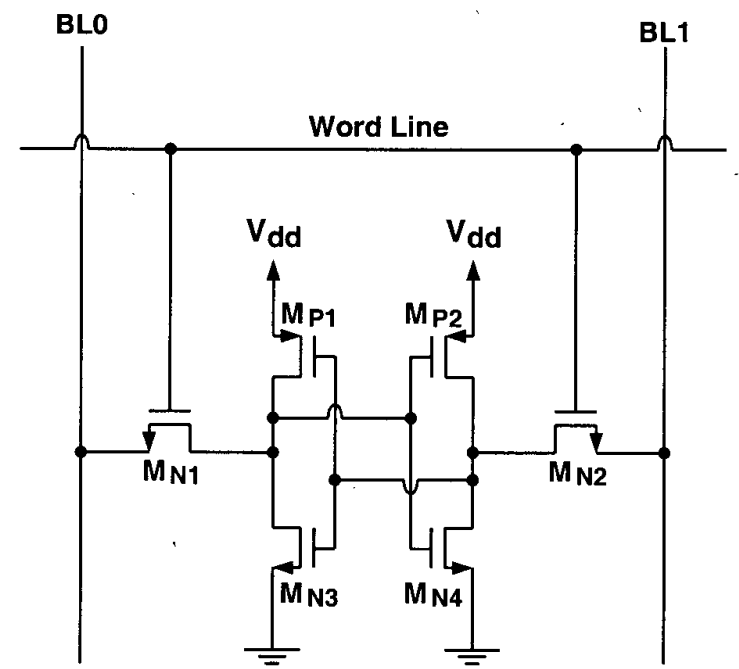

Figure 1. Structure of the conventional 6T SRAM cell.

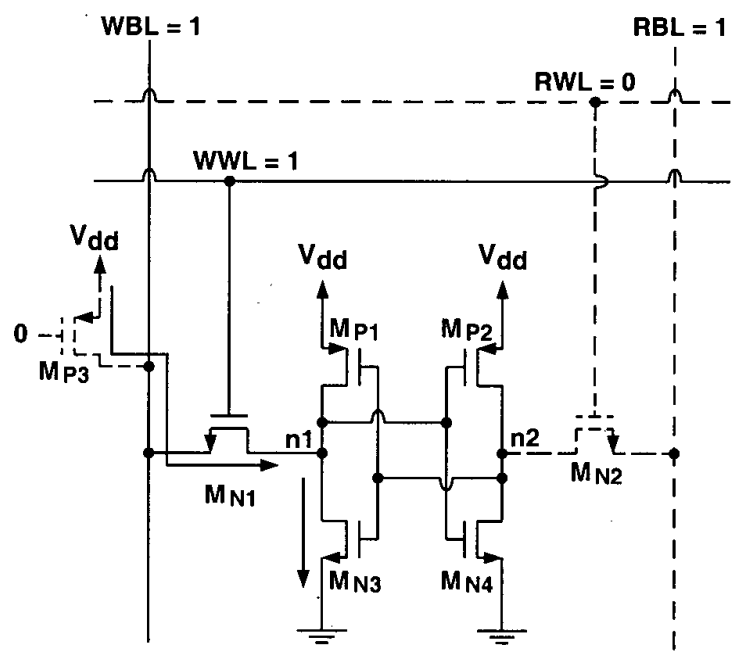

Figure 2. Single-bit-line write-1 operation of the conventional 6T SRAM cell.

is larger than the hole mobility of the PMOS device, the 
voltage of node $\mathrm{n} l$ is difficult to be raised during the singlebit-line write-logic-1 operation. As a result, the conventional SRAM cell structure cannot be used for two-port memory circuits with the single-bit-line write-logic-1 operation.

Although several techniques [1]-[2] have been applied to resolve this difficulty, the penalty cannot be justified for realizing two-port VLSI SRAM. Using DTMOS techniques, a two-port 6T SOI CMOS SRAM cell for low-voltage SRAM with SBLSRWA has been reported [3]. In this paper, with a unique structure by connecting the source terminal of an NMOS device in the SRAM cell to the write word line, this 6T SRAM cell can be used to provide single-bit-line simultaneous read-and-write access capability for $1 \mathrm{~V}$ twoport VLSI SRAM.

\section{SBLSRWA SRAM Cell}

Fig. 3 shows the single-bit-line simultaneous read-and-write access (SBLSRWA) two-port 6T SRAM cell. As shown in the figure, the source terminal of the NMOS device $M_{N 3}$ is connected to the write word line WWL instead of to the ground as in the conventional SRAM cell. In the SBLSRWA memory cell, the left side is connected to the write bit line WBL via the pass transistor $\mathrm{M}_{\mathrm{N} 1}$, which is controlled by write word line WWL. The right side of the SBLSRWA memory cell is connected to the read bit line RBL via the pass transistor $\mathrm{M}_{\mathrm{N} 2}$, which is controlled by the read word line RWL. By this configuration, simultaneous read and write accesses of the SBLSRWA can be facilitated. During the write-logic- 1 access, initially, logic- 0 is stored at node $n 1$. The previous problem associated with the turn-on of the

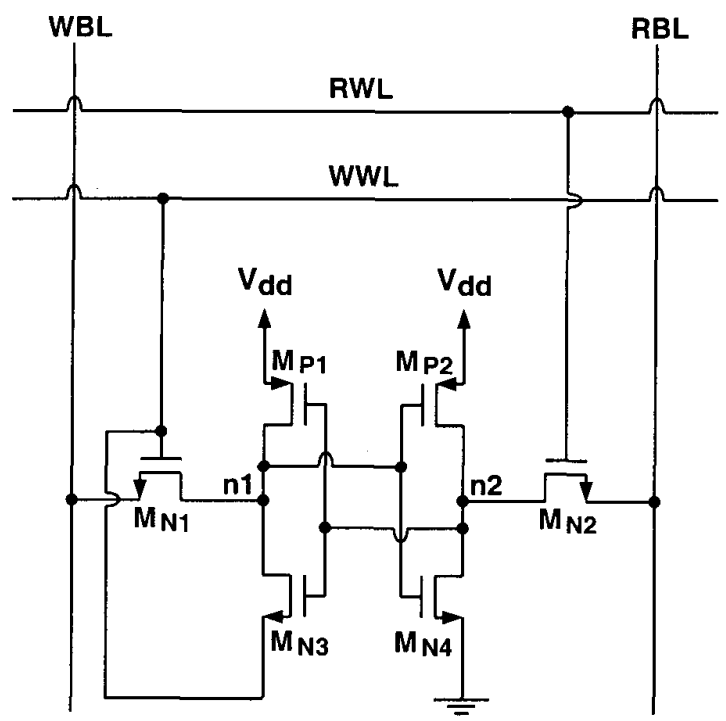

Figure 3. Structure of the two-port simultaneous read-andwrite access (SBLSRWA) 6T SRAM cell.
NMOS device $M_{N 3}$ in the conventional SRAM cell for single-bit-line write-logic-1 access can be avoided. Instead, owing to the write word line WWL-connected source terminal of $\mathrm{M}_{\mathrm{N} 3}$, during the single-bit-line write-logic-1 operation, the source of the NMOS device $\mathrm{M}_{\mathrm{N}}$ is tied to high, usually at $V_{d d}$. As a result, during the single-bit-line writelogic-1 operation, the voltage of node $n 1$ can reach high at $V_{d d}-V_{T N}$ easily. Consequently, $M_{N 4}$ turns on and $M_{P 2}$ turns off. Thus, the right side of the SBLSRWA cell, node $\mathrm{n} 2$, switches to $0 \mathrm{~V}$, which makes the voltage of node $\mathrm{n} l$ raised to 1V: This concludes the write-logic-1 operation.

In order to verify the effectiveness of the SBLSRWA SRAM cell, transient analysis during simultaneous read and write accesses of the SBLSRWA SRAM cell at a low supply voltage has been carried out. In the SBLSRWA SRAM cell under study, all six transistors have an aspect ratio of $0.3 \mu \mathrm{m} / 0.25 \mu \mathrm{m}$. Two parasitic capacitors of $0.1 \mathrm{pF}$ are assumed at write and read bit lines (WBL, RBL). Based on a $0.25 \mu \mathrm{m}$ CMOS technology, Fig. 4 shows the transient waveforms during the write access of the SBLSRWA $6 \mathrm{~T}$ SRAM cell at $\mathrm{V}_{\mathrm{dd}}$ of $1 \mathrm{~V}$ based on SPICE simulation results. As shown in the figure, there are four cases for the write access - (1) logic- 0 is written into the storage node $n 1$, which is stored with logic-0 (logic-0 $\rightarrow$ logic- 0 ), (2) logic-1 is written into the storage node $\mathrm{n} 1$, which is stored with logic-0 (logic-0 $\rightarrow$ logic-1), (3) logic-1 is written into the storage node $\mathrm{n} 1$, which is stored with logic-1 (logic-1 $\rightarrow$ logic-1), (4)logic-0 is written into the storage node $n 1$, which is stored with logic-1 (logic-1 $\rightarrow$ logic-0). The transients associated with these four cases are described below.

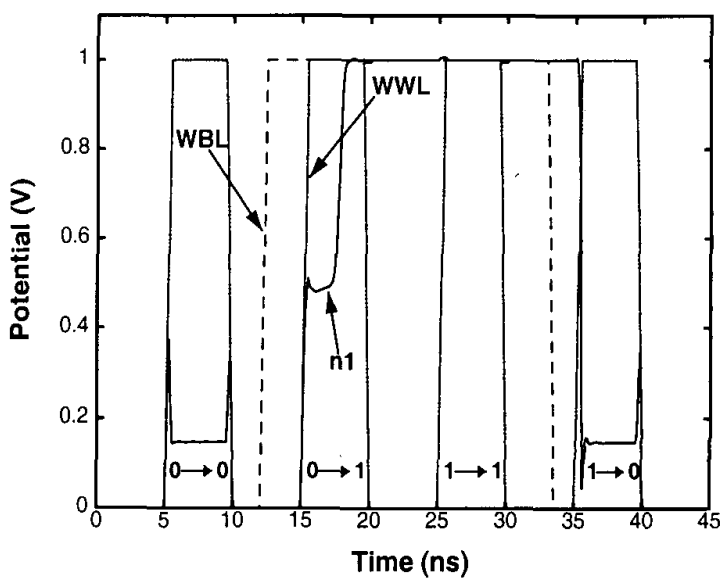

Figure. 4 Transients during the write access of the two-port SBLSRWA 6T SRAM cell at $\mathrm{V}_{\mathrm{dd}}$ of $1 \mathrm{~V}$.

\section{Logic-0 $\rightarrow$ Logic-0 Write}

In this case, before the single-bit-line write-logic-0 operation 
is executed (WWL $=0$ ), the storage node $n 1$ is at logic- $0(0 \mathrm{~V})$ since $\mathrm{M}_{\mathrm{N} 3}$ is on. The write bit line WBL is also at logic -0 $(0 \mathrm{~V})$. When WWL switches from low to high, write access is initiated. During the initial ramp-up period of WWL with its voltage before reaching the threshold voltage of $\mathrm{M}_{\mathrm{N} 1}\left(\mathrm{~V}_{\mathrm{TN}}^{\prime}\right)$, node $n 1$ is being charged with its voltage rising with WWL since $M_{N 3}$ is on. Note that at this time $M_{N 1}$ is off. During the ramp-up period of WWL with a voltage greater than the threshold voltage of $\mathrm{M}_{\mathrm{N} 1}\left(\mathrm{~V}_{\mathrm{TN}}\right), \mathrm{M}_{\mathrm{N} 1}$ turns on. At this time, since the write bit line is at logic-0, node $n 1$ is discharged as shown. Due to the ratioed-logic structure of $M_{N 1}$ and $M_{N 3}$, the voltage of node $\mathrm{nl}$ is maintained at $0.15 \mathrm{~V}$ during most of the write-logic- 0 access period when WWL is at its logic-1 value (1V). $\Lambda$ fter the write-logic-0 access is over, WWL ramps down. During the initial ramp-down of WWL, the transconductance of $\mathrm{M}_{\mathrm{Nl}}$ becomes smaller. As a result, despite the down-slew of WWL, duc to the ratioed-logic structure of $M_{N 1}$ and $M_{N 3}$, the voltage of node $n 1$ slcws upward during the initial ramp-down of WWL. When WWL is below $V_{T N}, M_{N 1}$ turns off and the voltage of node $n 1$ slews downward with the ramp-down of WWL. When WWL comes down to the logic-0 level $(0 \mathrm{~V})$, node $\mathrm{nl}$ is also pulled down to $0 \mathrm{~V}$. This accomplishes the $\operatorname{logic}-0 \rightarrow \operatorname{logic}-0$ write operation.

\section{Logic-0 $\rightarrow$ Logic-1 Write}

In this case, before the singlc-bit-line write-logic- 1 operation is executed (WWL $=0$ ), the storage node $\mathrm{nl}$ is at logic- $0(0 \mathrm{~V})$ since $M_{N 3}$ is on. The write bit line WBL is at logic-1 (IV). During the initial ramp-up period of WWL when WWL is smaller than $V_{T N}$, as in the logic- $0 \rightarrow \operatorname{logic}-0$ case, node $n l$ is being charged with its voltage rising with WWL since $M_{N 3}$ is on. When WWL is greater than $V_{T N}, M_{N 1}$ turns on. Different from the situation in the logic- $0 \rightarrow$ logic- 0 case, node $n 1$ rises further since WBL is now at $1 \mathrm{~V}$ instead of $0 \mathrm{~V}$. As a result, $\mathrm{M}_{\mathrm{NI}}$ also helps charging node $\mathrm{nl}$ toward $\mathrm{V}_{\mathrm{dcl}}$ $V_{\mathrm{TN}}$. The rise in node $\mathrm{n} I$ is coupled to node $\mathrm{n} 2$ - the gate of $\mathrm{M}_{\mathrm{N} 3}$ has a transient pulse with its peak greater than $\mathrm{V}_{\mathrm{dd}}$. Therefore, node $\mathrm{nl}$ also has a transicnt pulse with its peak exceeding $V_{\mathrm{dd}}-V_{\mathrm{TN}}$. Then, $\mathrm{M}_{\mathrm{P} 2}$ turns off and $\mathrm{M}_{\mathrm{N} 4}$ turns on - node $\mathrm{n} 2$ falls to $0 \mathrm{~V}$. Consequently, $\mathrm{M}_{\mathrm{Pl}}$ turns on and node $\mathrm{n} 1$ is pulled up to $\mathrm{V}_{\mathrm{dd}}$ of $1 \mathrm{~V}$ to conclude the write-logic-1 operation.

\section{Logic-1 $\rightarrow$ Logic-1 Write}

In this case, before the single-bit-line write-logic-1 operation is executed, $\mathrm{M}_{\mathrm{N} 3}$ is off and both WBL and node $\mathrm{nI}$ are at $1 \mathrm{~V}$. When WWL switches from low to high, $\mathrm{M}_{\mathrm{N} \text { I }}$ will not turn on because its $V_{G S}$ is equal to 0 . At this time, since both $M_{N 1}$ and $\mathrm{M}_{\mathrm{N} 3}$ are off, node $\mathrm{nl}$ maintains its logic- 1 potential stably throughout the whole write-logic-1 cyclc.

\section{Logic-1 $\rightarrow$ Logic-0 Write}

In this case, before the single-bit-line write-logic- 0 operation is executed, node $\mathrm{nl}$ is at logic- 1 (IV) since $\mathrm{M}_{\mathrm{N} 3}$ is off. Write bit line WBL is at logic-0 (0V). During the ramp-up of WWL above $V_{T N}, M_{N 1}$ turns on, thus node $n l$ is pulled down by the logic- 0 of WBL. As a result, $M_{N 4}$ turns off and $M_{p 2}$ turns on, which leads to the turn-off of $\mathrm{M}_{\mathrm{P}^{1} 1}$ and the turn-on of $\mathrm{M}_{\mathrm{N} 3}$. At this time, node $\mathrm{nl}$ is charged by $\mathrm{M}_{\mathrm{N} 3}$ and discharged by $\mathrm{M}_{\mathrm{Nl}}$. As a result, node $\mathrm{n} 1$ slews upward to a potential about $0.15 \mathrm{~V}$, which is determined by the ratioedlogic structure of $\mathrm{M}_{\mathrm{N} 1}$ and $\mathrm{M}_{\mathrm{N} 3}$ as described in the logic $-0 \rightarrow$ logic 0 case. When the write-logic -0 access is over, WWL ramps down. As in the logic- $0 \rightarrow \operatorname{logic}-0$ case, during the initial ramp-down of WWL, the transconductance of $\mathrm{M}_{\mathrm{N} 1}$ becomes smaller. As a result, despite the down-slew of WWL, due to the ratioed-logic structure of $\mathrm{M}_{\mathrm{N1}}$ and $\mathrm{M}_{\mathrm{N} 3}$, the voltage of node nl slews upward during the initial rampdown of WWL. When WWL, is below $V_{T N}, M_{N 1}$ turns off, and the voltage of node $\mathrm{I}$ slews downward with the rampdown of WWL. When WWL comes down to the logic-0 level $(0 \mathrm{~V})$, node $\mathrm{n} 1$ is also pulled down to $0 \mathrm{~V}$. This accomplishes the logic-1 $\rightarrow$ logic-0 write operation.

As shown in lig. 4, among four write cases, during the write pulse when WWI, is high, in both logic-0 $\rightarrow \operatorname{logic}-0$ and logic- $1 \rightarrow \operatorname{logic}-0$ cases, node $n 1$ is not at $0 \mathrm{~V}$. Instead, node $\mathrm{nl}$ is at $0.15 \mathrm{~V}$. This may consume transient power consumption, but can be overcome by shortening the pulse width of WWL.

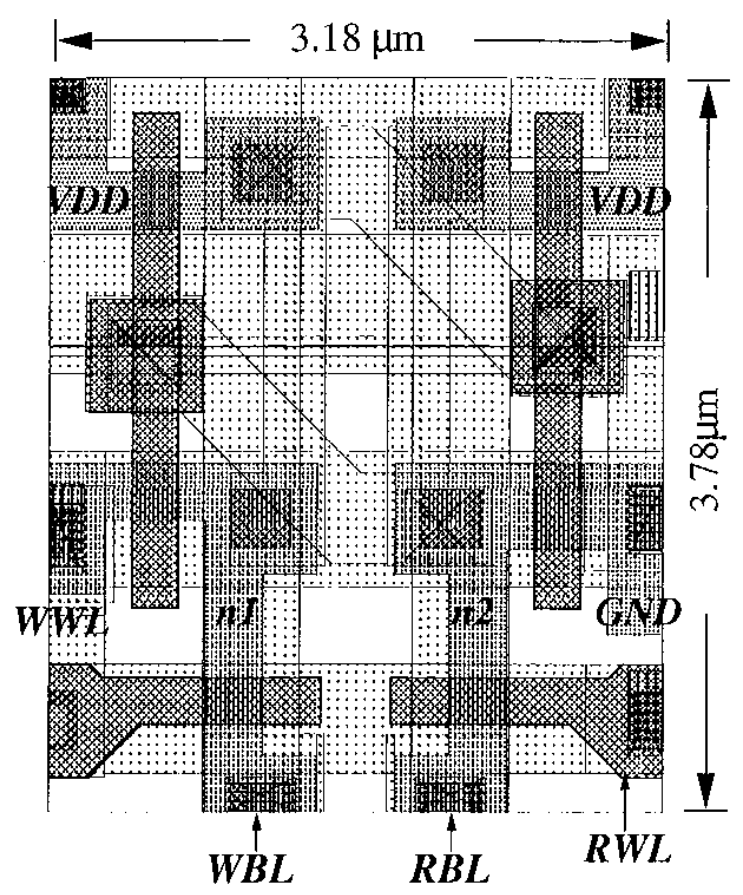

Figure 5. Layout of the SBLSRWA 6T SRAM cell. 
The SBLSRWA SRAM cell consists of six transistors and two bit lines. In contrast, in the conventional two-port $8 \mathrm{~T}$ SRAM cell, eight transistors and four bit lines are required. Thus, the SBLSRWA SRAM cell is much more compact as compared with the conventional two-port 8T SRAM cell. Something worth mentioning is that due to its asymmetrical configuration, the layout style of SBLSRWA 6T SRAM cell has to be different from that of the conventional 6T SRAM cell in order to minimize its area. In the conventional 6T SRAM cell (Fig. 1), the sources of NMOS devices $\mathrm{M}_{\mathrm{N} 3}$ and $\mathrm{M}_{\mathrm{N} 4}$ are both connected to ground, therefore they can share their layout regions to decrease the cell area. However, this technique cannot be applied to the SBLSRWA because the sources of $\mathrm{M}_{\mathrm{N} 3}$ and $\mathrm{M}_{\mathrm{N} 4}$ in it are no longer connected together. However, by sharing the source of $\mathrm{M}_{\mathrm{N} 3}$ with that of the cell at the left and the source of $\mathrm{M}_{\mathrm{N} 4}$ with that of the cell at the right, the layout area of the SBLSRWA 6T cell is almost the same as a conventional 6T SRAM cell. Fig. 5 shows the layout of the SBLSRWA 6T SRAM cell. Using a $0.25 \mu \mathrm{m}$ CMOS technology, its area is about $12 \mu \mathrm{m}^{2}$ and is much smaller than a conventional $8 \mathrm{~T}$ two-port SRAM cell whose area is approximately $20 \mu^{2}$ - a more than $40 \%$ reduction in the layout area of an SRAM cell.

\section{Conclusion}

In this paper, a two-port 6T CMOS SRAM cell structure for low-voltage VLSI SRAM with single-bit-line simultaneous read-and-write access (SBLSRWA) capability has been described. With a unique structure of connecting the source terminal of an NMOS device in the SRAM cell to the write word line, this SRAM cell can be used to provide SBLSRWA capability for IV two-port VLSI SRAM.

\section{References}

[1] T. S. Yang, M. A. Horowitz, and B. A. Wooley, " A 4ns 4Kxl bit Two-Port BiCMOS SRAM," IEEE Journal of Solid-State Cincuits, Vol. 23, Oct. 1988, pages 10301040.

[2] M. Ukita, S. Murakami, T. Yamagata, H. Kuriyama, Y. Nishimura, and K. Anami, "A Single-Bit-Line CrossPoint Cell Activation (SCPA) Architecture for UltraLow-Power SRAM's," IEEE Journal of Solid-State Circuits, Vol. 28, No. 11, Nov. 1993, pages 1114-1118.

[3] S. C. Liu and J. B. Kuo, " A Novel 0.7V Two-Port 6T SRAM Cell Structure with Single-Bit-Line Simultaneous Read-and-Write Access (SBLSRWA) Capability using PD SOI DTMOS Techniques," IEEE SOI Conference Dig., Sonoma County, CA, Oct 1999. 